\title{
Clustering, advection, and patterns in a model of population dynamics with neighborhood-dependent rates
}

\author{
Emilio Hernández-García ${ }^{1}$ and Cristóbal López ${ }^{1,2}$ \\ ${ }^{1}$ Instituto Mediterráneo de Estudios Avanzados IMEDEA (CSIC-UIB), Campus de la Universitat de les Illes Balears, \\ E-07122 Palma de Mallorca, Spain \\ ${ }^{2}$ Departament de Física, Universitat de les Illes Balears, E-07122 Palma de Mallorca, Spain
}

(Received 16 October 2003; published 30 July 2004)

\begin{abstract}
We introduce a simple model of population dynamics which considers reproducing individuals or particles with birth and death rates depending on the number of other individuals in their neighborhood. The model shows an inhomogeneous quasistationary pattern with many different clusters of particles arranged periodically in space. We derive the equation for the macroscopic density of particles, perform a linear stability analysis on it, and show that there is a finite-wavelength instability leading to pattern formation. This is responsible for the approximate periodicity with which the clusters of particles arrange in the microscopic model. In addition, we consider the population when immersed in a fluid medium and analyze the influence of advection on global properties of the model, such as the average number of individuals.
\end{abstract}

DOI: 10.1103/PhysRevE.70.016216

PACS number(s): 89.75.Kd, 87.23.Cc, 05.40.-a, 47.54.+r

\section{INTRODUCTION}

Interacting particle systems are useful models to understand a variety of effects in fields as diverse as condensed matter physics, chemical kinetics, population biology (where they are called individual based models) or sociology (agent based models) [1]. As one of the simplest examples one can consider an ensemble of Brownian particles, each one dying or duplicating with given probabilities per unit of time. Several authors [2-6] have considered such Brownian Bug (BB) model in the context of population dynamics (in particular to address plankton distributions and patchiness), in the case in which the probabilities of death and reproduction are equal. Aggregation of the particles in a decreasing number of clusters occurs. This clustering is somehow surprising since a standard mean-field or rate-equation description gives for the particle density $\rho$ the equation

$$
\frac{\partial \rho}{\partial t}=\left(\lambda_{0}-\beta_{0}\right) \rho+D \nabla^{2} \rho,
$$

where $D$ is the diffusion coefficient, and $\lambda_{0}$ and $\beta_{0}$ are the birth and death rates, respectively. Obviously, when $\lambda_{0}=\beta_{0}$, Eq. (1) is simply the diffusion equation which cannot lead to spatial inhomogeneities.

This result was known since some time ago for this and related models [2-9], and points out the relevance of the fluctuations present in the discrete stochastic particle model, neglected in a naive mean-field macroscopic description, and that lead to reproductive pair correlations: the mean rates of death and birth are equal, but if locally there is an excess of reproduction events, and diffusion is not fast enough, a cluster of particles will develop, whereas no birth will occur in empty zones and particles will simply disappear from regions with excess of death.

The authors of Ref. [2] go beyond that result, and show that the clustering persists even in the presence of rather strong stirring, as it would occur if the bugs live in a turbulent fluid such as the Ocean. The clusters now become elon- gated filaments, but there is still strong spatial inhomogeneity arising from the microscopic particle fluctuations and reproductive correlations.

The simple model just described misses some important features present in real biological populations. The most obvious is the absence of any interaction between the bugs. Among other consequences, the global dynamics of the system, i.e. the time evolution of the total number of particles, is completely independent of its spatial distribution. Thus stirring the system alters the spatial pattern of the bugs, but neither their individual lifetimes, nor the time history of the particle number, nor its statistical properties. In the context of aquatic biological populations this is known to be incorrect, as fluid stirring has strong impact on the population dynamics $[10,11]$.

In this paper we introduce interacting particle models by modifying the birth and death rates of the BB model. They will take into account the number of neighbors within a given distance of each bug. There is now a strong interplay between the bug dynamics and the ambient flow and, in addition, new effects arising from the spatial range of interaction occur and modify the reproductive-correlations clustering effect. In particular, an inhomogeneous steady structure with many different clusters of particles coming from different families (i.e., they are born from a different parent), and arranged in a periodic pattern, may occur. The number of particles in any of these clusters is similar, resembling the spreading of individuals in small groups over a geographical area. This pattern formation phenomenon occurs via a finite wavelength instability that can be characterized in a deterministic description, being fluctuations only of secondary importance. We analyze the phenomenon with a continuousfield Langevin description obtained from the particle model by Fock space techniques.

It turns out that, at the deterministic level, the continuousfield description coincides with the one recently discussed in Ref. [12] (see also Refs. [13-15]), a model for population dynamics of the Fisher-type where the dynamics of the popu- 
lation density is non-local. This approach generalizes the Fisher equation incorporating nonlocal effects in the quadratic term by means of an influence function. Biologically, this function may account for competition for resources within a certain space range, as for example, when nutrients have a large diffusivity. Given that the density equation for our discrete model is, in the absence of the noise term, exactly the one proposed in Ref. [12] for a particular form of the influence function, our work provides a possible justification at the level of microscopic particle dynamics of the emerging nonlocal nonlinear interactions in the density equations. In the last decade much interest has led to the study of other types of nonlocal models in ecological modeling. They are not directly related to our stochastic particle system, but they also describe interactions at a distance, most of them formulated in terms of continuous-field evolution equations for densities. Biologically, these interactions can be mediated through vision, hearing, smelling or other kinds of sensing. Aggregation of organisms is one of the topics widely researched through this line (see Ref. [16] for a review). In some cases the nonlocality arises from the type of motion of the individuals, such as in Ref. [17]. In others (more related to our particle model), it is via the production or annihilation process [18]. Typically the nonlocality appears as a linear integro-differential term. The models in Refs. [12-15] represent a different class, closer to our approach, in which nonlocality is in the nonlinear interactions. At variance with the present work, however, they do not consider the demographic fluctuations inherent to stochastic particle models. As shown later, this effect does not alter the pattern forming process, but leads to shifting in extinction points, absorbing transitions, and distinct behavior of the mean density as a function of parameters, among other consequences.

The paper is organized as follows. In Sec. II we introduce the discrete models. In Sec. III we study numerically some of their properties. In Sec. IV we study the pattern formation process and perform a stability analysis within the continuum-field description of our model. In Sec. V we study the influence of a fluid flow on the particle system, and in the last section we summarize our Conclusions. The Appendix contains the derivation of the continuum-field representation of our particle model, performed via Fock space techniques (see [19] for the application of the same techniques also in the context of population dynamics).

\section{MODELS}

In this section we introduce the discrete models that are the subject of study of the paper. We begin defining the original BB model and then our extensions.

\section{A. BB model}

The microscopic rules are simply enumerated [2]. Let $N(t)$ the number of bugs in the system (a two-dimensional periodic box of size $L \times L$; in all our computer simulations we will take $L=1$ ):

(1) There is an initial population of $N(t=0)=N_{0}$ bugs or particles, randomly located.
(2) One particle is selected at random and it dies with probability $p$, reproduces with probability $q$, or remains unchanged with probability $r(p+q+r=1)$. In the case of reproduction, the newborn particle is located at the same place as the parent particle. The process is repeated a number $N(t)$ of times [20].

(3) Each particle moves in random direction a distance drawn from a Gaussian distribution of standard deviation $\sigma$ (this models Brownian motion).

(4) When advection is considered, the particles are transported by an external flow to be described later.

(5) Time is incremented an amount $\tau$, and the algorithm repeats.

Symbolically, in chemical reaction notation:

$$
\begin{gathered}
\stackrel{\beta_{0}}{A \rightarrow} \varnothing, \\
\stackrel{\lambda_{0}}{A \rightarrow A+A,}
\end{gathered}
$$

where $A$ represents individual particles, each one dying at a rate $\beta_{0}=p / \tau$ (death rate per particle and unit of time), or reproducing at a rate $\lambda_{0}=q / \tau$. The Brownian motion step leads to diffusion with a diffusion coefficient $D=\sigma^{2} / 2 \tau$. In the following we measure time in units of $\tau$, so that $\tau=1$, $\beta_{0}=p, \lambda_{0}=q$, and $\sigma=\sqrt{2 D}$. We take $r=0$ so that $\lambda_{0}+\beta_{0}=1$, and define the important parameter $\mu=\lambda_{0}-\beta_{0}$, the difference between birth and death rates.

\section{B. Neighbourhood-dependent (ND) model}

The new model is analogous to the one before, except that in step 2 the reproduction and death rates of a given particle labelled $j, \lambda(j)$ and $\beta(j)$, are not constant but depend on the number of particles surrounding the particle $j$. Explicitly we take (with $\tau=1$ ):

$$
\lambda(j)=\max \left(0, \lambda_{0}-\frac{1}{N_{s}} N_{R}^{j}\right),
$$

and

$$
\beta(j)=\max \left(0, \beta_{0}-\frac{\alpha}{N_{s}} N_{R}^{j}\right),
$$

where $N_{R}^{j}$ denotes the total number of particles which are at a distance smaller than $R$ from particle $j$ (excluding the particle $j$ itself). $R$ is thus a range of interaction, $N_{s}$ is a saturation parameter, and $\alpha$ controls the asymmetry between its influence on death and on reproduction. The BB model is recovered when $R \rightarrow 0$. The maximum condition is imposed to insure that $\lambda(j)$ and $\beta(j)$ are positive definite, as it should be given that they are probabilities. When $N_{s}$ is positive, the model penalizes reproduction when particles are crowdedly surrounded. Lonely particles reproduce with higher probability. This kind of interaction would be appropriate to model individuals that compete for resources (e.g., food), in a neighborhood of its actual position. When $\alpha / N_{s}$ is positive, death rate decreases in crowded environments, modeling a kind of mutual protection. The opposite behavior occurs when these parameters are negative, a situation that will not 
be considered in the present paper, though most of the results presented can be extended easily to this case. This model of interacting particles is related to many others (see reviews in Refs. [1,21]) in which some limitation in the growth of the population at a single site is imposed via a fermionic restriction (explicitly stated [22] or implicitly imposed on computer simulations by forbidding double occupation of lattice sites) or via the inclusion of the coagulation process $A+A \rightarrow A$ inverse to $(3)[23,24]$. Our model (and the BB model) shares with them several qualitative features, the most important being the fact that the empty state is an absorbing state: if at some moment all the population becomes extinct, no recovery is possible within the rules of the model. This leads to an absorbing phase transition from an active or surviving phase to an absorbing dead phase when some effective reproduction rate is reduced. The peculiarity in our model is that interactions are not purely local, but extend to a finite distance $R$. We expect this to be irrelevant for the critical behavior close to the absorbing phase transition, since only asymptotically large scales are important there, and then we believe that this transition in our model will be in the standard Directed Percolation (DP) or Reggeon field-theory universality class to which many of these interacting models belong $[21,25]$. We will see, however, that the behavior in the active phase is greatly influenced by the existence of a finite interaction range $R$. In consequence we will not analyze in great detail the absorbing phase transition, but concentrate in the active phase(s), where more novel behavior occurs.

\section{NUMERICAL STUDY OF THE DISCRETE MODELS}

This section is devoted to presenting some numerical results that stress the differences between the BB and the ND model. Here we consider the system with no external driving flow, whose analysis is left to Sec. V.

The BB model has been studied in detail [2-5]. If $\mu=\lambda_{0}$ $-\beta_{0}>0$, the total population generally explodes exponentially, with a time scale given by $\mu^{-1}$, although there is a finite probability for extinction that depends on the initial population and decreases with increasing $\mu$. If $\mu<0$ the final state is, with probability 1 , the total extinction of the particles, occurring again at a exponential rate characterized on average by $\mu^{-1}$, but with diverging relative fluctuations. A critical situation occurs when $\lambda_{0}=\beta_{0}$. In this case, the particles arising from the same ancestor form clusters, with the number of clusters decreasing in time and the number of particles in the surviving clusters growing, so that $\langle N(t)\rangle$ $=N_{0} \forall t$, with the average taken over different realizations. But fluctuations in $N(t)$ are huge (its variance diverges linearly in time), with some runs leading to fast extinctions, and others with clusters surviving for long time. In a finite system all clusters finally disappear, but the typical lifetime diverges linearly with $N_{0}$, and the average lifetime is infinity [5]. Figure 1 shows the distribution of particles at two different stages of the evolution, one in which a large single cluster, coming from a single ancestor, is present (right panel) corresponding to a long-time evolution, and another with still many clusters from different ancestors (left panel)
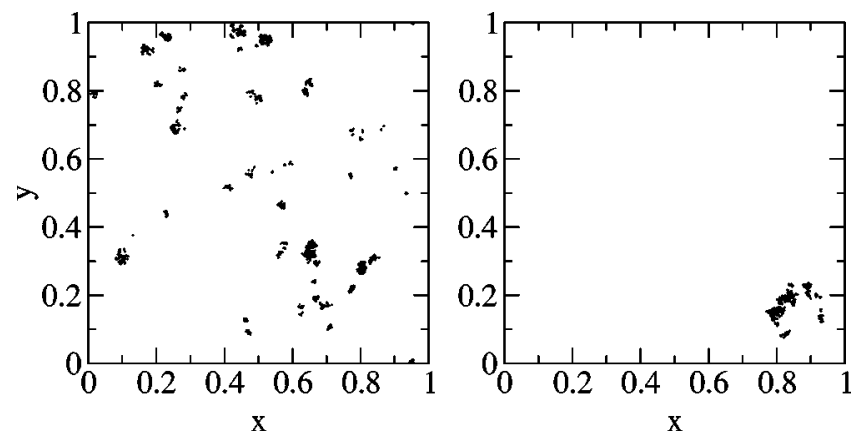

FIG. 1. Spatial configurations for the BB model at two different times. Left: configuration after 100 steps, with a large number of surviving clusters. Right corresponds to a single cluster remaining after 3000 steps. The value of the parameters are $\lambda_{0}=\beta_{0}=0.5, D$ $=10^{-5}$, and the initial population is of $N_{0}=1500$ bugs randomly distributed.

for an earlier stage of the temporal evolution.

Figure 2(a) shows the time evolution of the total number of bugs $N(t)$ in the critical case, $\mu=0$, for a particular realization, displaying the critical fluctuations, and examples of cases with nonvanishing $\mu$. One can observe the fast decay (growth) of $N(t)$ for $\mu$ negative (positive).

The behavior of the ND model is rather different. Just for simplicity we consider here (and in the rest of the paper) the value $\alpha=0$ so that only reproduction depends on the neighborhood. Figure 2(b) shows the time evolution of the population. For $\mu$ smaller than a critical value $\mu_{c}>0$ (which turns out to be $\mu_{c} \approx 0.4$ for the parameter values used in the figure), we find always extinction, whereas typical realizations reach a finite average population at long times for $\mu>\mu_{c}$. We plot in Fig. 3 the total average number of particles $N(t)$ at long-time vs $\mu$, and different values of the parameters. The scaling used to present $N(t)$ is suggested from an analytical expression discussed in next section. As discussed later, it

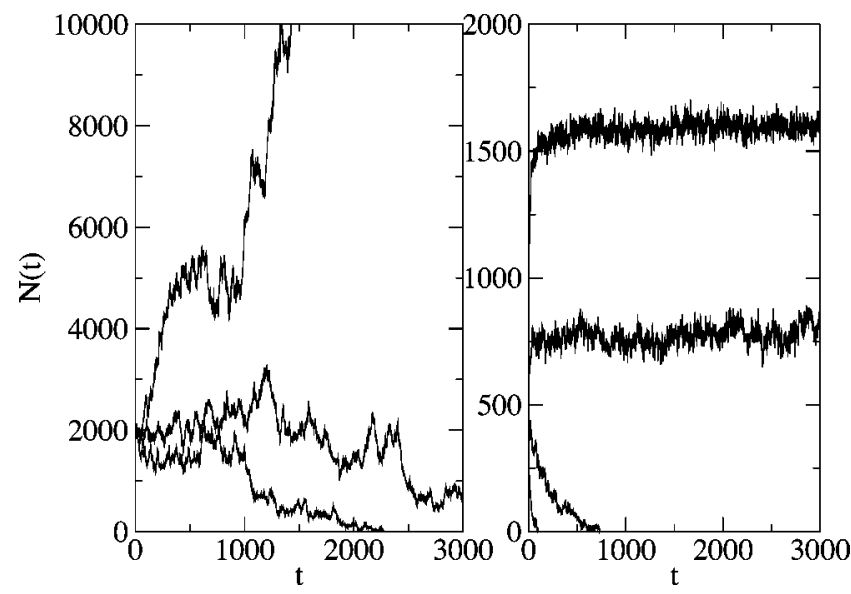

FIG. 2. (a) Total number of particles, $N(t)$, vs time for the BB model and three different values of the control parameter $\mu$ : From top to bottom: $\mu=5 \times 10^{-4}, \mu=0$, and $\mu=-5 \times 10^{-4}$; here $D=10^{-5}$. (b) Idem for the ND model and four different values of $\mu$ : From top to bottom, $\mu=0.7, \mu=0.5, \mu=0.4$, and $\mu=0.3$. Two are above critical $\left(\mu_{c} \approx 0.4\right)$ and two below it. The other parameter values are $R$ $=0.1, N_{s}=50$, and $D=10^{-5}$. 

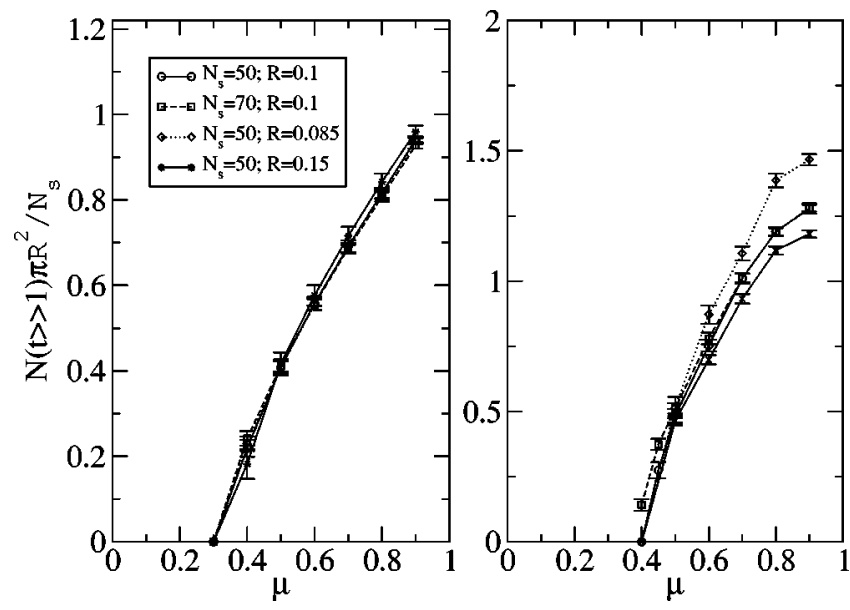

FIG. 3. Long-time average number of particles $N(t)$ vs $\mu$. Left panel corresponds to $D=10^{-4}$ and right to $D=10^{-5}$, and the other parameters as indicated. The average is taken from the instantaneous particle numbers at times between 1000 and 10000 steps; the error bar indicates the standard deviation of the instantaneous fluctuations around this mean value.

provides good data collapse in the left plot Fig. 3, for a diffusion coefficient of $D=10^{-4}$, but it is grossly inadequate for data in the right panel, corresponding to smaller diffusivity, $D=10^{-5}$.

The nature of the spatial distribution in the active phase depends on the values of the parameters. For large enough $D$, the spatial distribution of particles is homogeneous on average, whereas clear clustering occurs for small $D$. As in the BB model, the clusters are coming from different families. But here they are not transient and the most striking feature is that they organize in a periodic pattern. The periodicity of the pattern is of the order of $R$, the interaction range. In addition to decreasing $D$, this transition to a periodic organization occurs by increasing $R$ and, for small enough $D$, by increasing $\mu$. Figure 4 shows examples of the different spatial patterns.

We see that the most notable effect of the introduction of interactions with a characteristic spatial scale has been the segregation of bugs in a periodic array of clusters (bottomright plot in Fig. 4). This seem to be a rather natural way to make compatible the high local growth at relatively large value of $\mu$, with the reduction of this growth that a too crowded neighborhood would imply: the empty space between the clusters acts as a buffer zone keeping the competition for resources less limiting than in a homogeneous distribution. We expect that this mechanism will appear in Nature when there is a scattering of the total population in small groups over a large spatial area. One can think, for instance, of the spreading of groups of predators, or even of primitive human societies that are aggregated in small tribes.

We characterize the patterns in terms of the structure factor $S(K)$. It is defined as

$$
S(K)=\left\langle\left|\frac{1}{N(t)} \sum_{j} e^{i \mathbf{q} \cdot \mathbf{x}_{j}(t)}\right|^{2}\right\rangle_{K, t},
$$

where $\mathbf{x}_{j}(t)=\left(x_{j}, y_{j}\right)$ is the position vector of the particle $j$ at time $t, \mathbf{q}=\left(q_{x}, q_{y}\right)$ is a two-dimensional wave vector, the sum
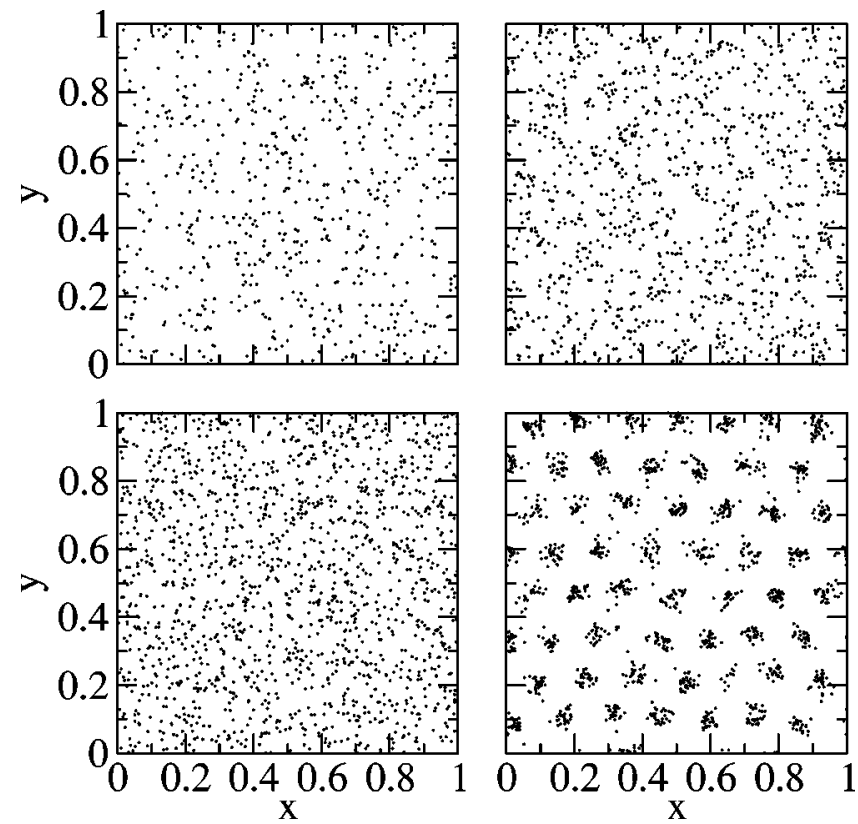

FIG. 4. Long-time spatial structures for the ND model. Left column corresponds to two patterns with the same value of $D$ $=10^{-4}$, and two different values of $\mu=0.5$ (up) and $\mu=0.9$ (bottom). Right column corresponds to fixed $\mu=0.7$, and $D=10^{-4}$ (upper), and $D=10^{-5}$ (bottom). In all the plots, $N_{s}=50$ and $R=0.1$.

is over all particles, and the average is a spherical average over all wave vectors of modulus $|\mathbf{q}|=K$, and a further temporal average in the long-time state is added to improve statistics. Maxima in this function identify relevant periodicities in the interparticle distribution.

In Fig. 5 we show the structure factor in the steady state of the model for different values of the parameters. The emergence of the periodic patterns is indicated by the peak in the structure factor. As in Fig. 4, we show here two different scenarios: The upper panels correspond to the structure factor (left) of different patterns with $\mu$ fixed and changing $D$.
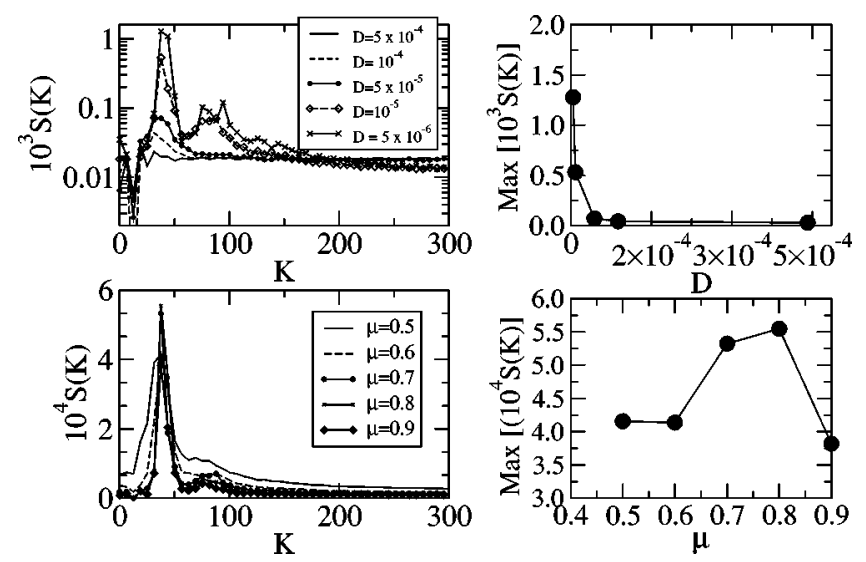

FIG. 5. Structure factors (left panels) and corresponding height of the main peak (right) for different patterns in the ND model. Upper panels are for fixed value of $\mu=0.7$ and the values of $D$ shown in the legend box. Bottom panels are for $D=10^{-5}$ and different $\mu$ 's as shown in the legend box. In all the plots, $N_{s}=50$ and $R=0.1$. Note the different scales in all the plots. 
One can observe that by decreasing $D$ the value of the peak increases (upper-right panel), indicating that clustering with a strong periodicity develops. Bottom panels are for $D$ $=10^{-5}$ and different values of $\mu$. Here the pattern is rather developed at all values of $\mu$ above the absorbing transition at $\mu_{c} \approx 0.4$, with only mild variations of the peak height (right panel) with $\mu$. By analyzing nonspherically-averaged versions of (6), we confirm that the periodic pattern has hexagonal symmetry at onset.

We next try to explain quantitatively the observed patterns in terms of an analytical description.

\section{FIELD THEORY DESCRIPTION, STABILITY ANALYSIS, AND SPATIAL PATTERNS}

Standard theories and tools for pattern formation studies [26] address continuous field models, and are not particularly well suited to analyze pattern formation in particle systems. Fortunately, there are well established techniques (known under the name of Doi-Peliti theory or Fock space techniques $[8,27,28])$ that allow a description of interacting particle systems in terms of field-theoretic Langevin equations. These techniques turn out to be equivalent to the Poisson representation $[29,30]$. In the simplest cases, the interacting particles are instantaneously Poisson distributed in each small space region, and the field description gives the space and time varying average value $\phi(\mathbf{x}, t)$ of the local Poisson distribution for the particle density $\rho$.

In general, however, the continuous field is complex and this simple interpretation does not hold, but still in this case all the moments of the particle density $\rho$ can be obtained from the moments of the fluctuating field $\phi$. For example the first moment of both quantities are equal $\langle\rho(\mathbf{x}, t)\rangle=\langle\phi(\mathbf{x}, t)\rangle$. In the Appendix, we derive in detail the Langevin field description for the ND model. Two approximations are needed to arrive to the final form (A20) (with an additional diffusion term, see the Appendix for details). As a first attack to the problem of pattern formation in our particle model, we analyze in this section just the deterministic part of the field equation, i.e., the noise term will be neglected. We will see that this will be sufficient to understand the main qualitative features of the pattern forming instability. The expected influence of the noise would be to affect system properties in the vicinity of transitions and instabilities, and to shift the position of the transition lines [25]. In addition, since our system is translational invariant and two-dimensional, it is very likely that the sharp bifurcation to patterns that we find in the deterministic analysis will be blurred by noise into a nonsharp crossover even in the thermodynamic limit. Nevertheless, we find good qualitative agreement between several of the observed properties of the discrete model presented in the preceding section and the deterministic predictions obtained here.

Thus we analyze the deterministic version of Eq. (A20) (with the diffusion term added and $\alpha=0$ ):

$$
\begin{aligned}
\partial_{t} \phi(\mathbf{x}, t)= & D \nabla^{2} \phi(\mathbf{x}, t)+\left(\lambda_{0}-\beta_{0}\right) \phi(\mathbf{x}, t) \\
& -\frac{1}{N_{s}} \phi(\mathbf{x}, t) \int_{|\mathbf{x}-\mathbf{r}|<R} d \mathbf{r} \phi(\mathbf{r}, t) .
\end{aligned}
$$

This equation is related to the one in Refs. [12,13,15] but

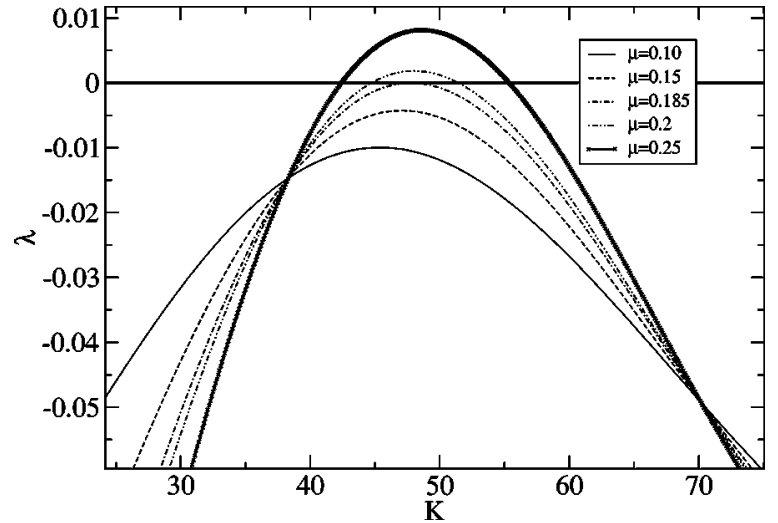

FIG. 6. Linear growth rate $\lambda$ vs wave number $K$ from (8) for different values of $\mu$ close to $\mu_{P}$. We take $R=0.1$ and $D=10^{-5}$ so that $\mu_{P}=0.185$ and $K_{m}=47.79$.

with a particular integral kernel. At this level of mean-field like approximation (no noise), the field $\phi(\mathbf{x}, t)$ can be interpreted as the density field $\rho(\mathbf{x}, t)$. Stationary homogenous solutions of this equation are the absorbing phase $\phi(\mathbf{x}, t)$ $=0$, and the active or survival phase $\phi(\mathbf{x}, t)=\phi_{s}=\mu N_{s} / \pi R^{2}$ (remember that $\mu=\lambda_{0}-\beta_{0}$ ). For $\mu<0$ the only stable solution is the absorbing one; the transition to the survival state is approached at $\mu=0$, and this state is stable for a range of positive values of $\mu$. At the deterministic level the transition is transcritical. As expected, particle fluctuations in the discrete model change this transcritical character of the transition (see Fig. 3), probably to one of the DP-type [21,25], occurring at values of $\mu$ larger than zero.

As mentioned before, Eq. (7) corresponds to the model in Ref. [12] if an influence function $f_{R}(\mathbf{r}, t)$ of the form $f_{R}(\mathbf{r}, t)=1$ if $|\mathbf{r}| \leqslant R, f_{R}(\mathbf{r}, t)=0$ if $|\mathbf{r}| \geqslant R$ is taken. Two limits arise in (7): when $R$ goes to zero, the Fisher equation with local interaction arises, where no patterns appear. The opposite limit, $R$ taking the value of the size of the system, has been considered in Ref. [12], and the nonexistence of spatial structures has been reported. Thus, in this work we limit to the study of intermediate values of $R$.

We make a stability analysis of the $\phi_{s}$ solution by considering small harmonic perturbations around it, $\phi(\mathbf{x}, t)=\phi_{s}$ $+\delta \phi(\mathbf{x}, t)$, with $\delta \phi(\mathbf{x}, t) \propto \exp (\lambda t+i \mathbf{k} \cdot \mathbf{x})$. After simple calculations one arrives at the following dispersion relation:

$$
\lambda(K)=-D K^{2}-\frac{2 \mu}{K R} J_{1}(K R),
$$

where $K$ is the modulus of $\mathbf{k}$, and $J_{1}$ is the first-order Bessel function. It is clear that the relevant parameters in the problem are $\mu$ and $D . / R^{2}$ (in fact the precise adimensional combinations are $\mu \tau$ and $D \tau / R^{2}$, but remember that we are measuring times in units of $\tau$, so that $\tau=1)$. The eigenvalue $\lambda(K)$ (which is in fact a function of $K R, \mu$, and $D / R^{2}$ ) is real and can be positive for some values of the parameters. This is shown in Fig. 6, where we plot $\lambda$ against $K$ for different values of $\mu$ around $\mu_{P}$ as given below in Eq. (13), with fixed $D / R^{2}$.

The equations 


$$
\begin{gathered}
\left.\frac{\partial \lambda(K)}{\partial K}\right|_{K=K_{m}}=0, \\
\lambda\left(K_{m}\right)=0,
\end{gathered}
$$

identify the values of the parameters at which the maximum of the curve $\lambda(K)$, at $K_{m}$, becomes positive. This gives a line of instability $\mu_{P}=\mu_{P}\left(D / R^{2}\right)$ in the parameter plane. It is straightforward to obtain that $\mu_{P}=-D R K_{m}^{3} /\left(2 J_{1}\left(K_{m} R\right)\right)$ and the equation for $K_{m}$ reads

$$
\frac{K_{m} R}{2 J_{1}\left(K_{m} R\right)}\left(J_{0}\left(K_{m} R\right)-J_{2}\left(K_{m} R\right)\right)-3=0 .
$$

$J_{0}$ and $J_{2}$ are the zero and second order Bessel functions, respectively. This equation can be solved numerically to obtain

$$
K_{m} \approx \frac{4.779}{R}
$$

so that

$$
\mu_{P} \approx 185.192 \frac{D}{R^{2}} .
$$

The behavior of the deterministic Eq. (7) is now clear: for $\mu<0$ the only stable solution is $\phi=0$. In the interval $0<\mu<\mu_{P}$ one has the homogeneous density $\phi=\phi_{s}$, and for $\mu>\mu_{P}$ spatial patterns emerge. This last transition can also be crossed by decreasing $D / R^{2}$ at fixed $\mu>0$.

This scenario is consistent with the results for the particle ND model shown in Sec. II. In particular, note that Eq. (12) indicates that the pattern periodicity is determined by $R$, and is independent of other parameters of the system such as $\mu$, $D$, and $N_{s}$. This is in agreement with the results for the structure factors shown in Fig. 5. It is also observed there that the numerical value of the dominant wave number in Fig. 5 is close to the predicted value given by Eq. (12). Since Eq. (7) has no particular symmetries, we expect on generic grounds [26] that hexagonal patterns would appear close to the instability. Since they usually bifurcate subcritically we expect some range of bistability for $\mu<\mu_{P}$, that may be influenced by noise. In consequence we do not expect the transition line (13) to be fully accurate. Nevertheless it correctly explains the distinct behavior between the data shown in Fig. 3(a) (essentially all of them predicted to be in the homogeneous phase, as confirmed by Figs. 4 and 5) and Fig. 3(b) (for smaller $D$, so that all data points are in the periodic clustered phase). The curves in Fig. 3(a) collapse together and approach the deterministic prediction for the homogeneous solution $\phi_{s}$ (a straight line of slope 1 in that scaled plot) sufficiently far from the absorbing transition point. Such collapse does not occur in Fig. 3(b) since they do not correspond to homogeneous states. More important are the fluctuation corrections to our deterministic results around the absorbing transition: the transition point is quite far from the deterministic value $\mu_{c}=0$ and the critical behavior is very different from the simple linear vanishing of the number of particles predicted deterministically (transcritical bifurcation).

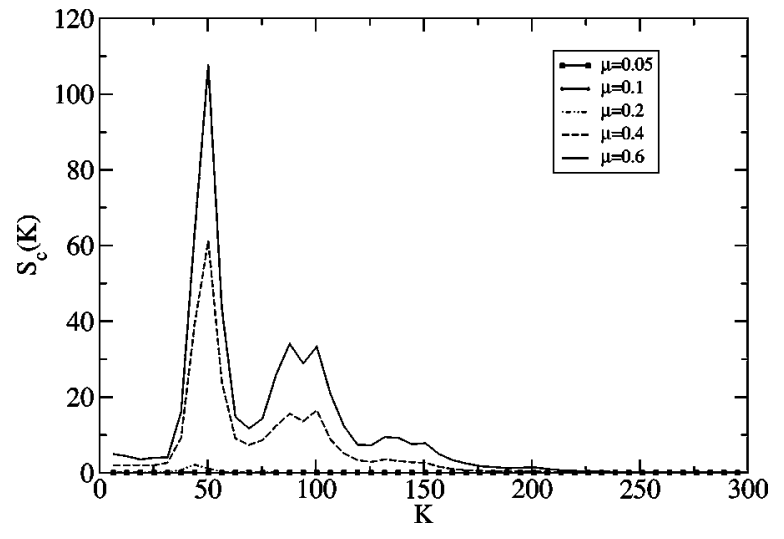

FIG. 7. Spherically averaged continuum structure function against $K$ for different values of the control parameter $\mu$. The other parameter values are $D=10^{-5}$ and $R=0.1$.

In Fig. 7 we plot the spherically averaged structure function, $S_{c}(K)$, against the wave number, $K$, of the density field $\phi$ obtained numerically, after a long-time, from numerical solution of Eq. (7). $S_{c}(K)$ is the modulus of the spatial Fourier transform of $\phi(\mathbf{x}, t)$, averaged spherically and in time. Note that, since $\phi$ is a continuous field, $S_{c}(K)$ is related but not identical to the structure factor $S(K)$ of the particle system, Eq. (6). Nevertheless, maxima of $S_{c}(K)$ also identify dominant periodicities. In Fig. 7 we have taken $R=0.1, D$ $=10^{-5}$, so that $\mu_{P} \approx 0.185$. One can see how for $\mu>\mu_{P}$ the structure function develops a peak that grows with $\mu$, indicating the development of a spatial pattern with a typical distance between clusters. The peak is located at the wave number closest to (12) compatible with the discretization imposed by the periodic boundary conditions. Figure 8 shows a steady pattern of density which is analogous to the one shown for the discrete model in the bottom-right panel of Fig. 4. These observations confirm for the full nonlinear model (7) the behavior identified from the linear stability analysis of the homogeneous solutions. It is also worth men-

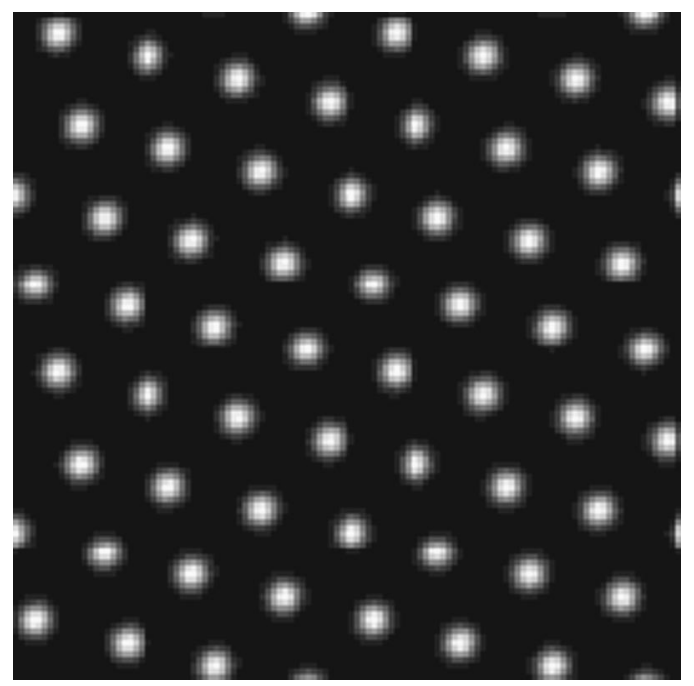

FIG. 8. Steady spatial pattern from the deterministic equation (7). $\mu=0.70, R=0.1, D=10^{-5}$, and $N_{s}=50$. Note the strong similarity with the pattern in the bottom-right plot in Fig. 4. 
tioning that the nonspherically-averaged version of the structure function displays hexagonal order for $\mu \geq \mu_{P}$. Transitions to other geometries (squares) seem to occur at high values of $\mu$, but we have not pursued the issue further (square geometry appears for a different integral kernel in Ref. [12]).

\section{INFLUENCE OF FLUID FLOW}

In addition to the pattern-forming instability, a crucial difference between the BB and the neighborhood-dependent model is their response to an external flow. Since the birth and death rates of the BB model are fixed constants, global quantities such as the total number of particles are independent of any particle motion, being it diffusive or hydrodynamic. This seems to be rather unrealistic for applications such as modeling plankton populations [2,10,11], always driven by external flows. On the contrary, with the neighborhood dependence of the rates in the tribal model we overpass this inconvenience and the model becomes dependent on the environmental conditions.

As a simple illustration of the impact of a velocity field, we consider the flow given by the Harper map (the one also used in Ref. [2]), which is a symplectic map in two dimensions and, therefore, it resembles an incompressible flow. At each time step the fourth item in the algorithm described in Sec. II consists of moving the particles in the following way: If we denote by $\left(x_{i}(t), y_{i}(t)\right)$ the coordinates of the particle $i$ at time $t$, after one iteration of the map they become

$$
\begin{gathered}
x_{i}\left(t^{\prime}\right)=x_{i}(t)+A \cos \left(y_{i}(t)\right), \\
y_{i}\left(t^{\prime}\right)=y_{i}(t)+A \cos \left(x_{i}\left(t^{\prime}\right)\right),
\end{gathered}
$$

where $t^{\prime}=t+\tau$. A gives the strength of the flow, and depending of its value particles can follow regular or stochastic trajectories. To check for the robustness of the results with respect to the particular flow considered, we have also implemented advection by another widely used model to study chaotic advection by incompressible fluid flows, the Standard map [31]:

$$
\begin{gathered}
x_{i}\left(t^{\prime}\right)=x_{i}(t)+\frac{A}{2 \pi} \sin \left(2 \pi y_{i}(t)\right), \\
y_{i}\left(t^{\prime}\right)=y_{i}(t)+x_{i}\left(t^{\prime}\right) .
\end{gathered}
$$

Here we are just interested in highlighting the behavior when the flow changes, that is when $A$ changes. Increasing $A$ leads to increasing irregularity and mixing behavior in both flows. The time evolution of the total number of particles is given in Fig. 9 (the left plot corresponds to the Harper map flow and the right one to the Standard map). It is seen that the asymptotic value depends on the flow strength $A$. The reason can be clearly seen from Fig. 10, where snapshots of the particle distributions are presented for the Harper case. It is seen that the periodic array of clusters in the absence of flow becomes more filamental-like as the flow strength increases. The shape of the filamental structures reflects the known unstable and stable foliation of phase space for the Harper map.
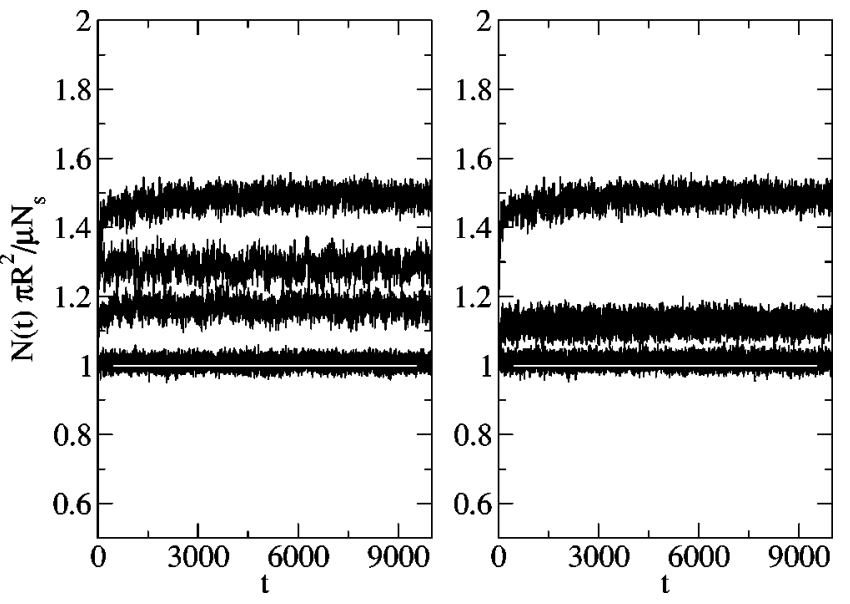

FIG. 9. $N(t)$, normalized with $\mu N_{s} / \pi R^{2}$, vs time for different values of the external flow strength, $A$, and the two chaotic maps in the text. Left is for the Harper map and right for the Standard map. From top to bottom: $A=0, A=0.01, A=0.05$, and, fluctuating around the value 1 (white line), $A=1$ and $A=3$. The $A=0.01$ and $A=0.05$ plots cannot be properly distinguished for the figure of the Standard map. The other parameters: $\mu=0.8, N_{s}=50, D=10^{-5}$, and $R=0.1$.

As for the BB model [2], inhomogeneity persists for rather strong flow, but finally the distribution becomes homogeneized. At this point, the particle distribution should be very close to Poissonian, with density given by the homogeneous solution of (7). This is indeed what is observed in the figure (for large values of $A$ the total number of particles, $N(t)$, normalized with the survival steady density value, fluctuates around 1). For smaller flow strength $A$ the spatial structure in the neighborhood of each particle becomes relevant, and the number of particles approaches the value in the absence of
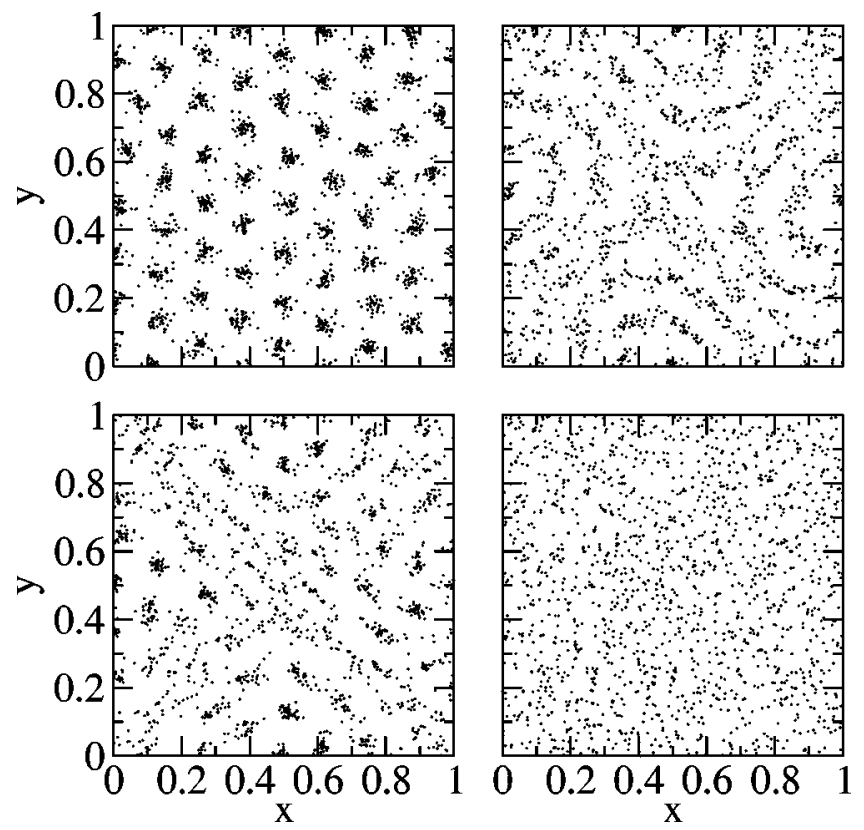

FIG. 10. Long-time spatial structure for the ND model with an external flow (Harper map). From top to bottom and left to right, $A=0, A=0.1, A=0.5$, and $A=1$. The other parameters: $\mu=0.9, D$ $=5 \times 10^{-6}, N_{s}=50$, and $R=0.1$. 


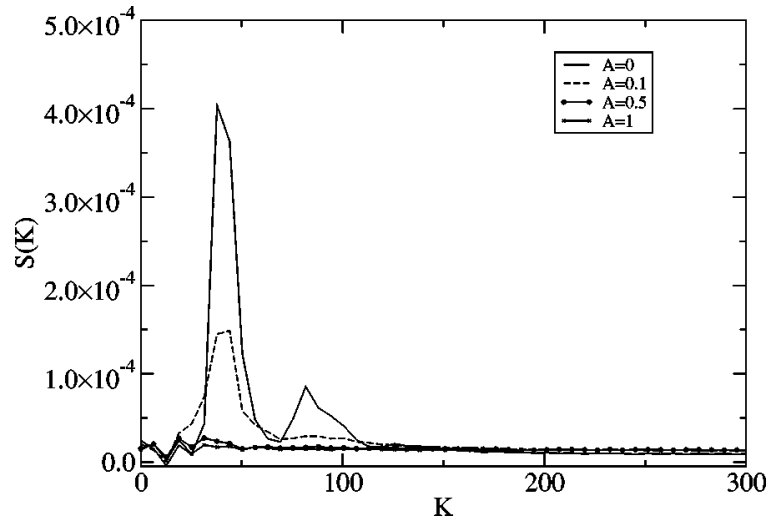

FIG. 11. Structure factor for the patterns shown in Fig. 10.

flow, given in Fig. 3. For completeness, Fig. 11 shows, in terms of the structure factor, the disappearance of structure as the flow strength increases. Thus, we see that the nonlocal interactions lead to a coupling between flow and population dynamics, mediated by the changes in local distribution geometry that the flow induces.

\section{SUMMARY}

In this paper we have introduced an interacting particles model which considers birth and death rates for any individual depending on the number of particles that are within a distance smaller than a given range of interaction. The model can be considered as a simple nonlocal interacting extension of the BB model, but showing, however, a very different behavior. A striking feature is the appearance of a quasistationary (fluctuations are present) periodic pattern of particle clusters.

In order to deepen in the understanding of the pattern forming instabilities and structures in the discrete model, we have derived the continuum equation describing the particle distribution. Two main features characterize this equation: (a) the presence in the deterministic part of an integral term taking into account the nonlocal interaction among the particles, and (b) the very complex structure of the noise term, which also reflects the nonlocality of the interaction. To understand the pattern forming process we have studied the deterministic part of the equation. It coincides with other models previously studied in the literature [12]. At this level, the interpretation of the continuum field as the density field of particles is valid. Periodic clustering appears as a finite wavelength instability in the density equation.

In addition, we have also considered the response of the model to an external driving flow. Global properties of the system like the total number of particles depend on the flow, via the influence of it in the spatial structure.

Future extensions of this work will include a further study of the instabilities, an explicit consideration of the noise term in the continuum description, and the consideration of different (chemical or biological) species of particles interacting through a finite interaction range.

\section{ACKNOWLEDGMENTS}

We acknowledge discussions with Guido Boffetta, Stefano Musacchio, and Angelo Vulpiani. We also acknowledge support from MCyT of Spain and FEDER under Project Nos. REN2001-0802-C02-01/MAR (IMAGEN) and BFM20001108 (CONOCE).

\section{APPENDIX: FIELD THEORETICAL APPROACH}

In this appendix we derive an approximate continuum field equation describing the dynamics of our model, by using Fock space techniques. We present more results than needed for the deterministic analysis of Sec. IV, but we include them here by its intrinsic interest and to highlight the presence of particle fluctuations in the original discrete model.

The standard formulation of Fock space methods $[8,28]$ requires the microscopic model to be defined on a lattice. Thus we first modify our off-lattice model to fit into a grid, apply the standard procedure, and then let the grid spacing to become negligibly small, so that we recover the continuum off-lattice limit. This is somehow different from a macroscopic limit, since only the grid size, but not the basic lengths in our model, such as the interaction range $R$, will be made to vanish. From the lattice approximation we can write the stochastic dynamics in terms of a Hamiltonian operator. A path integral representation can then be obtained and a Langevin equation can be derived from it. The off-lattice limit is then conveniently taken. The whole process cannot be performed exactly and we need to introduce two approximations, one valid for not too large particle densities and another restricting to Gaussian fluctuations.

We divide space in cells forming a lattice of $\Lambda$ sites and describe the state of the system by the number of particles inside each lattice cell $\left\{N_{i}\right\}_{i=1, \ldots, \Lambda}$. The lattice model will be equivalent to the off-lattice one in the limit of small lattice size, so that only zero or one particles will be present at each site. In addition it is convenient to work in continuous time, so that a continuous time Markovian Master Equation describes the dynamics. At long times $(\gg \tau)$ there should be no difference between the discrete and the continuous time approaches.

Since statistically independent processes are represented by additive terms in the Master equation, we can treat separately particle diffusion and particle transformations (we do not consider advection here, since it is not used in the analysis of Sec. IV), and then sum up the corresponding contributions. It is well known $[8,28]$ that the random walk process leads to a diffusion term in the continuous Langevin equation, and no contribution to the noise term. This will not be rederived here. We focus in the birth and death of particles:

At each site we have a birth rate per particle

$$
\lambda_{i}=\max \left(0, \lambda_{0}-\frac{1}{N_{s}} \sum_{j \in R(i)} N_{j}\right)
$$

and a death rate per particle

$$
\beta_{i}=\max \left(0, \beta_{0}-\frac{\alpha}{N_{s}} \sum_{j \in R(i)} N_{j}\right) .
$$

The sum is over the cells that are within a distance $R$ from the site $i$ (excluding cell $i$, so that in the continuum limit we 
recover the original prescriptions (4) and (5)). Defining $P\left(N_{1}, N_{2}, \ldots, N_{\Lambda} ; t\right)=P(\{N\} ; t)$ as the probability of having $N_{1}$ bugs in site $1, \ldots, N_{\Lambda}$ bugs in site $\Lambda$ at time $t$, we have the following Master Equation:

$$
\begin{aligned}
\frac{d P(\{N\} ; t)}{d t}= & \sum_{i=1}^{\Lambda}\left(N_{i}-1\right) \lambda_{i} P\left(\ldots, N_{i}-1, \cdots ; t\right) \\
& +\sum_{i=1}^{\Lambda}\left(N_{i}+1\right) \beta_{i} P\left(\ldots, N_{i}+1, \cdots ; t\right) \\
& -\sum_{i=1}^{\Lambda} N_{i} \lambda_{i} P\left(\ldots, N_{i}, \cdots ; t\right) \\
& -\sum_{i=1}^{\Lambda} N_{i} \beta_{i} P\left(\ldots, N_{i}, \ldots ; t\right) .
\end{aligned}
$$

The Fock space representation starts by defining the many-particle state vector:

$$
|\psi\rangle=\sum_{N_{1} \ldots N_{\Lambda}} P(\{N\} ; t) \prod_{i=1}^{\Lambda}\left(a_{i}^{\dagger}\right)^{N_{i}}|0\rangle,
$$

where $a_{i}^{\dagger}$ and $a_{i}$ are the creation and annihilation operators at lattice site $i$, that is,

$$
\begin{gathered}
a_{i}^{\dagger}\left|N_{1} \cdots N_{i} \cdots N_{\Lambda}\right\rangle=\left|N_{1} \cdots N_{i}+1 \cdots N_{\Lambda}\right\rangle, \\
a_{i}\left|N_{1} \cdots N_{i} \cdots N_{\Lambda}\right\rangle=N_{i}\left|N_{1} \cdots N_{i}-1 \cdots N_{\Lambda}\right\rangle,
\end{gathered}
$$

verifying bosonic commutation rules

$$
\begin{gathered}
{\left[a_{i}, a_{j}^{\dagger}\right]=\delta_{i j},} \\
a_{i}|0\rangle=0 .
\end{gathered}
$$

Thus we have:

$$
\begin{aligned}
\frac{d|\psi\rangle}{d t}= & \sum_{\{N\}} \frac{d P(\{N\} ; t)}{d t} \prod_{i=1}^{\Lambda}\left(a_{i}^{\dagger}\right)^{N_{i} \mid}|0\rangle \\
= & \sum_{\{N\}, i}\left[\left(N_{i}-1\right) \lambda_{i} P\left(\cdots N_{i}-1 \cdots\right)\right. \\
& \left.\times \prod_{i=1}^{\Lambda}\left(a_{i}^{\dagger}\right)^{N_{i} \mid} 0\right\rangle-N_{i} \lambda_{i} P\left(\cdots N_{i} \cdots\right) \\
& \times \prod_{i=1}^{\Lambda}\left(a_{i}^{\dagger}\right)^{N_{i} \mid}|0\rangle+\left(N_{i}+1\right) \beta_{i} P\left(N_{i}+1\right) \\
& \times \prod_{i=1}^{\Lambda}\left(a_{i}^{\dagger}\right)^{N_{i}|0\rangle-N_{i} \beta_{i} P\left(\cdots N_{i} \cdots\right)} \\
& \left.\times \prod_{i=1}^{\Lambda}\left(a_{i}^{\dagger}\right)^{N_{i}|0\rangle}\right] .
\end{aligned}
$$

After simple algebra we have a Schrödinger-type equation in Euclidean time:

$$
\frac{d|\psi\rangle}{d t}=-H|\psi\rangle
$$

with the Hamiltonian:

$$
H\left(a_{i}^{\dagger}, a_{i}\right)=-\sum_{i}\left(\left[\left(a_{i}^{\dagger}\right)^{2} a_{i}-a_{i}^{\dagger} a_{i}\right]{\hat{\lambda_{i}}}_{i}\left[a_{i}-a_{i}^{\dagger} a_{i}\right] \hat{\beta}_{i}\right) .
$$

$\hat{\lambda}_{i}$ and $\hat{\beta}_{i}$ are the operator versions of $\lambda_{i}$ and $\beta_{i}$, that is, expressions (A1) and (A2) with all the particle numbers $N_{k}$ replaced by the number operators $a_{k}^{\dagger} a_{k}$. They can be defined from a power series representation [32] of a conveniently regularized version of expressions (A1) and (A2).

To obtain a path integral representation, a first step is to use the commutation relations in $H$ until obtaining normal ordering (i.e., creation operators to the left and annihilation to the right). We call the resulting expression $H_{\mathrm{NO}}$. Then an action depending on the classical complex variables $\psi_{i}^{*}(t)$ and $\psi_{i}(t)$ is computed as:

$$
S=\int_{0}^{t} d t \sum_{i}\left[\psi_{i}^{*} \partial_{t} \psi_{i}+H_{\mathrm{NO}}\left(a_{i}^{\dagger} \rightarrow \psi_{i}^{*}+1, a_{i} \rightarrow \psi_{i}\right)\right] .
$$

The "arrow" notation means that the operators $a_{i}^{\dagger}$ and $a_{i}$ should be replaced by the indicated classical variables. The action allows to calculate average of lattice quantities such as $N_{i}$ as adequate path integrals over $\psi_{i}$ and $\psi_{i}^{*}$ involving the weight $e^{-S}$. For Hamiltonian (A11), it turns out that normal ordering leads to intricate expressions in any regularized version of (A1) and (A2). We note that for low enough densities (or large $N_{s}$ ), the maximum condition would be rarely needed. In consequence, a sensible approximation at low density is:

$$
\hat{\lambda_{i}} \approx \lambda_{0}-\frac{1}{N_{s j}} \sum_{j \in R(i)} a_{j}^{\dagger} a_{j}
$$

and

$$
\hat{\beta}_{i} \approx \beta_{0}-\frac{\alpha}{N_{s}} \sum_{j \in R(i)} a_{j}^{\dagger} a_{j}
$$

In regions where densities are not small, these expressions would need corrections. We will comment more on this later.

The fact that $\hat{\lambda}_{i}$ and $\hat{\beta}_{i}$ do not depend on bosonic operators on site $i$ (the sum $j \in R(i)$ excludes the cell $i$ ) makes trivial the normal-ordering procedure. The action is

$$
\begin{aligned}
S= & \int_{0}^{t} d t \sum_{i}\left\{\psi_{i}^{*}\left[\partial_{t} \psi_{i}+\left(\beta_{0}-\lambda_{0}\right) \psi_{i}+\frac{1-\alpha}{N_{s}} \psi_{i} \sum_{j \in R(i)} \psi_{j}\right]\right. \\
& +\left(\psi_{i}^{*}\right)^{2}\left[-\lambda_{0} \psi_{i}+\frac{1}{N_{s}} \psi_{i} \sum_{j \in R(i)} \psi_{j}\right]+\frac{1-\alpha}{N_{s}}\left|\psi_{i}\right|^{2} \sum_{j \in R(i)}\left|\psi_{j}\right|^{2} \\
& \left.+\frac{1}{N_{s}}\left|\psi_{i}\right|^{2} \psi_{i}^{*} \sum_{j \in R(i)}\left|\psi_{j}\right|^{2}\right\} .
\end{aligned}
$$

An extra term (not written) should be added to implement the particular initial condition used for $P(\{N\}, t)$. One could 
approach the continuum limit at this step but, for clarity, we will perform it after writing down the Langevin equation. By introducing Gaussian noises at each site, $\left\{\eta_{k}(t)\right\}$, as Gaussian integrals in averages involving Eq. (A15), one realizes that averages of physical quantities, such as $\left\langle N_{k}\right\rangle$ can be obtained as averages over stochastic processes $\left\{\psi_{k}(t)\right\}$ satisfying Îto stochastic differential equations. Terms in the action linear in $\left\{\psi_{k}^{*}\right\}$ give rise to deterministic terms in the stochastic equation, terms quadratic in these variables determine the correlations of the Gaussian noises $\left\{\eta_{k}(t)\right\}$. Terms of higher order give rise to non-Gaussian noise statistics. As usual we neglect these terms (this is our second approximation, by which we neglect the last term in (A15)) and obtain an approximate Îto Langevin equation:

$$
\partial_{t} \psi_{i}(t)=\left(\lambda_{0}-\beta_{0}\right) \psi_{i}-\frac{1-\alpha}{N_{s}} \psi_{i} \sum_{j \in\langle R(i)\rangle} \psi_{j}+\eta_{i}(t)
$$

where the Gaussian noises $\left\{\eta_{k}(t)\right\}$ obey:

$$
\begin{gathered}
\left\langle\eta_{i}\right\rangle=0, \\
\left\langle\eta_{i}(t) \eta_{j}\left(t^{\prime}\right)\right\rangle=2 \delta\left(t-t^{\prime}\right)\left\{\delta_{i j}\left[\left(\lambda_{0}-\frac{1}{N_{s j}} \sum_{j(i)} \psi_{j}\right) \psi_{i}\right]\right. \\
\left.-\theta_{i j}(R) \frac{1-\alpha}{N_{s}} \psi_{i} \psi_{j}\right\} .
\end{gathered}
$$

$\theta_{i j}(R)$ is a function with value 1 if cells $i$ and $j$ are different and each one is within a distance $R$ from the other. Noise correlations are multiplicative, nonlocal, and rather involved. In addition, they cannot be satisfied by real stochastic process $\left\{\eta_{k}(t)\right\}$ so that the noise terms, and the variables $\left\{\psi_{k}(t)\right\}$, are in general complex valued. Despite this, the particle statistics is encoded in the lattice stochastic process. For example, the probability for the occupation numbers is given by [29]

$$
P(\{N\}, t)=\left\langle\prod_{i=1}^{\Lambda} \frac{e^{-\psi_{i}(t)} \psi_{i}(t)^{N_{i}}}{N_{i} !}\right\rangle_{\left\{\psi_{i}(t)\right\}},
$$

where the average is over the statistics of the processes $\left\{\psi_{i}\right\}$. Consequences of (A19) are $\left\langle N_{i}(t)\right\rangle=\left\langle\psi_{i}(t)\right\rangle$ and $\left\langle N_{i}(t)^{2}\right\rangle$ $=\left\langle\psi_{i}(t)^{2}\right\rangle+\left\langle\psi_{i}(t)\right\rangle$. The term dependent on the initial condition that was omitted from (A15) can now be taken into account by providing adequate initial conditions for $\left\{\psi_{k}(t)\right\}$, linked to the initial particle statistics by (A19).
The off-lattice continuum limit is performed by introducing the density $\phi(\mathbf{x}, t)$ by the change $\psi_{i} \rightarrow \phi(\mathbf{x}, t) \Delta^{d}$ and taking the lattice spacing going to zero $\Delta \rightarrow 0 . d$ is the spatial dimension ( $d=2$ through this paper). We have some freedom in performing this limit. For example, if we take the interaction range $R$ to be a fixed number of lattice spacings, then $R \rightarrow 0$ in the continuum limit. In this case non-locality is lost, and by properly scaling the interaction parameters $N_{s}$ and $\alpha$, we get a continuous equation related to Reggeon field theory that has been thoroughly studied [21,25]. But we want to describe the macroscopic behavior of the off-lattice models introduced in Sec. II, in which the interaction range is finite. Thus, we fix $R$ to a finite value when going to the continuum and obtain

$$
\begin{aligned}
\partial_{t} \phi(\mathbf{x}, t)= & \left(\lambda_{0}-\beta_{0}\right) \phi(\mathbf{x}, t)-\frac{1-\alpha}{N_{s}} \phi(\mathbf{x}, t) \int_{|\mathbf{x}-\mathbf{r}|<R} d \mathbf{r} \phi(\mathbf{r}, t) \\
& +\eta(\mathbf{x}, t),
\end{aligned}
$$

with

$$
\begin{aligned}
&\langle\eta(\mathbf{x}, t)\rangle=0, \\
&\left\langle\eta(\mathbf{x}, t) \eta\left(\mathbf{x}^{\prime}, t^{\prime}\right)\right\rangle= 2 \delta\left(t-t^{\prime}\right)\left\{\delta\left(\mathbf{x}-\mathbf{x}^{\prime}\right) \phi(\mathbf{x}, t)\right. \\
& \times\left[\lambda_{0}-\frac{1}{N_{s}} \int_{0<|\mathbf{x}-\mathbf{r}|<R} d \mathbf{r} \phi(\mathbf{r}, t)\right] \\
&\left.-\frac{1-\alpha}{N_{s}} \phi(\mathbf{x}, t) \phi\left(\mathbf{x}^{\prime}, t\right) \theta_{\mathbf{x x}^{\prime}}(R)\right\} .
\end{aligned}
$$

The continuum description of the BB model is recovered if $R \rightarrow 0$. As in the discrete case, correlations in (A22) are multiplicative, nonlocal, and lead to complex valued processes. The diffusion term $D \nabla^{2} \phi$ should also be included in the r.h.s. of (A20) to account for the random walk of the particles.

We see that the deterministic part of (A20) with the diffusion term is what one would guess as a mean-field description for the particle density when taking into account the finite range of the interactions, and it has been obtained here by a more systematic approach. Since we expect the simple mean field approach to be correct for high enough densities and far from transition points, we conclude that the terms neglected in the low density approximation (A13) and (A14) are just correcting fluctuation statistics in a regime, high densities, in which they are already not very relevant.
[1] J. Marro and R. Dickman, Nonequilibrium Phase Transitions in Lattice Models (Cambridge University Press, Cambridge, 1998).

[2] W. R. Young, A. J. Roberts, and G. Stuhne, Nature (London) 412, 328 (2001); W. R. Young, in Proceedings of the 12th
'Aha Huliko'a Hawaiian Winter Workshop (University of Hawaii, Manoa, 2001).

[3] R. Adler, in Proceedings of the 10th 'Aha Huliko'a Hawaiian Winter Workshop (University of Hawaii, Manoa, 1997).

[4] A. M. Etheridge, An Introduction to Superprocesses (American 
Mathematical Society, New York, 2000).

[5] Y-C. Zhang, M. Serva, and M. Polikarpov, J. Stat. Phys. 58, 849 (1990).

[6] B. Houchmandzadeh, Phys. Rev. E 66, 052902 (2002).

[7] M. Meyer, S. Havlin, and A. Bunde, Phys. Rev. E 54, 5567 (1996).

[8] J. Cardy, in The Mathematical Beauty of Physics, edited by J. M. Drouffe and J. B. Zuber (World Scientific, Singapore, 1996).

[9] N. M. Shnerb, Y. Louzoun, E. Bettelheim, and S. Solomon, Proc. Natl. Acad. Sci. U.S.A. 97, 10322 (2000).

[10] A. P. Martin, K. J. Richards, A. Provenzale, and A. Bracco, Global Biogeochem. Cycles 16:10.1029/2001GB001449 (2002).

[11] A. P. Martin, Prog. Oceanogr. 57, 125 (2003).

[12] M. A. Fuentes, M. N. Kuperman, and V. M. Kenkre, Phys. Rev. Lett. 91, 158104 (2003).

[13] A. Sasaki, J. Theor. Biol. 186, 415 (1997).

[14] H. Sayama, M. A. M. de Aguiar, Y. Bar-Yam, and M. Baranger, Phys. Rev. E 65, 051919 (2002).

[15] J. Billingham, Nonlinearity 17, 313 (2004).

[16] G. Flierl, D. Grunbaum, S. Levin, and D. Olson, J. Theor. Biol. 196, 397 (1999).

[17] A. Mogilner and L. Edelstein-Keshet, J. Math. Biol. 38, 534 (1999); A. Mogilner, L. Edelstein-Keshet, L. Bent, and A. Spiros, ibid. 47, 353 (2003).

[18] J. L. Boldrini et al., J. Theor. Biol. 185, 523 (1997); L. Allen and E. J. Allen, Bull. Math. Biol. 58, 815 (1996).

[19] C. Escudero, J. Buceta, F. J. de la Rubia, and Katja Lindenberg, Phys. Rev. E 69, 021908 (2004).

[20] This is asynchronous updating. In this simple BB model, the order of the updating (synchronous, asynchronous, sequential) is irrelevant, but it is important in the neighborhood dependent models.

[21] H. Hinrichsen, Adv. Phys. 49, 815 (2000).

[22] E. Moro, Phys. Rev. E 68, 025102(R) (2003).

[23] J. Cardy and U. C. Täuber, Phys. Rev. Lett. 77, 4780 (1996); J. Stat. Phys. 90, 1 (1998).

[24] J. Kockelkoren and H. Chaté, Phys. Rev. Lett. 90, 125701 (2003).

[25] G. Grinstein and M. A. Muñoz, in Fourth Granada Lectures in Computational Physics, edited by P. L. Garrido and J. Marro (Springer, Berlin, 1997).

[26] M. C. Cross and P. C. Hohenberg, Rev. Mod. Phys. 65, 851 (1993).

[27] M. Doi, J. Phys. A 9, 1465 (1979); L. Peliti, J. Phys. (France) 46, 1469 (1985).

[28] The following references contain short and useful summaries of the technique: L. Pechenik and H. Levine, Phys. Rev. E 59, 3893 (1999); E. Bettelheim, O. Agam, and N. M. Shnerb, Physica E (Amsterdam) 9, 600 (2001); A. Kamenev, in Strongly Correlated Fermions and Bosons in LowDimensional Disordered Systems, NATO Science Series II, Vol. 72, edited by I. V. Lerner, B. L. Altshuler, V. I. Falko, and T. Giamarchi (Kluwer, Dordrecht, 2002).

[29] C. W. Gardiner, Handbook of Stochastic Methods for Physics, Chemistry, and the Natural Sciences (Springer, New York, 1985).

[30] M. Droz and A. McKane, J. Phys. A 27, L467 (1994).

[31] E. Ott, Chaos in Dynamical Systems (Cambridge University Press, Cambridge, 1993).

[32] J. Zinn-Justin, Quantum Field Theory and Critical Phenomena (Oxford University Press, Oxford, 1997). 